\title{
Acute Myocardial Infarction Manifested with Headache
}

\author{
Yiannis S. Chatzizisis*, Panagiotis Saravakos, Amalia Boufidou, Despoina Parharidou and \\ Ioannis Styliadis
}

$1^{\text {st }}$ Cardiology Department, AHEPA University Hospital, Aristotle University Medical School, Thessaloniki, Greece

\begin{abstract}
We report a very rare case of a patient who presented with headache as the sole symptom of an acute myocardial infarction (AMI). The patient underwent primary percutaneous coronary angioplasty followed by drug-eluting stent implantation and the headache was immediately relieved. The pathophysiologic explanation of the occurrence of headache as a sole manifestation of an AMI is discussed.
\end{abstract}

Keywords: Acute myocardial infarction, headache.

\section{INTRODUCTION}

Despite the progress in the laboratory detection of acute myocardial infarction (AMI), medical history remains the most important diagnostic step in order to establish the diagnosis. Most patients with AMI describe a severe, pressuretype pain in the mid-sternum, often radiating to the left arm, neck or jaw [1]. The pain resembles angina, but it can be distinguished from it by its intensity, duration ( $>30 \mathrm{~min}$ ), and failure to resolve with nitroglycerin administration. The pain may also be accompanied by nausea, vomiting and diarrhea, especially when the infarction is located in the inferior wall [1]. Other symptoms include dyspnea, diaphoresis, dizziness, palpitations, cold perspiration, profound weakness and syncope $[1,2]$. The diagnosis of AMI may be difficult when atypical symptoms occur, such as indigestion, unusual localization of the pain, agitation and altered mental status $[1,2]$. Furthermore, AMI may be silent in more than $25 \%$ of cases, in which the infarction is not recognized by the patient and evidence of the infarction is provided by the electrocardiogram or post-mortem examination. Asymptomatic infarction occurs more frequently in elderly patients with hypertension, angina pectoris, and, mostly, diabetes mellitus, as a result of the polyneuropathy that accompanies long-standing and uncontrolled diabetes [1].

We describe a case presented with headache as the sole symptom of AMI.

\section{CASE REPORT}

A 42-year-old male, with no history of coronary artery disease or any other risk factor, including hypertension, hyperlipidemia, smoking, and positive family history, presented to a primary health center, complaining for acute onset of severe headache beginning $4 \mathrm{~h}$ before. The headache was located mainly frontally and bitemporally and was

*Address correspondence to this author at the $1^{\text {st }}$ Cardiology Department AHEPA University Hospital Aristotle University Medical School 1 Stylp. Kyriakidi Street, Thessaloniki, 54636, Greece; Tel: +30 2310 994837; Fax: +30 2310 994837; E-mail: joc@med.auth.gr constant with no periods of relief, even after taking analgesics. Since the physical examination and electrocardiogram were normal (Fig. 1a), no further evaluation was requested and the patient was discharged home. Two hours later, the patient was admitted to our emergency department complaining of persistent headache. On admission, he was conscious, well-orientated and the vital signs and physical examination were normal. The electrocardiogram showed a $\mathrm{q}$ wave with mild ST elevation $(1.2 \mathrm{~mm})$ and inverted $\mathrm{T}$ wave in the precordial leads $V_{2}-V_{5}$ (Fig. 1b). Laboratory examination revealed the following abnormal findings: serum glucose $382 \mathrm{mg} / \mathrm{dl}$ (70-110 mg/dl), CK $336 \mathrm{U} / \mathrm{I}$ (0-190 U/I), CK-MB 32 U/l (0-24 U/I), LDH 459 U/I (240-410 U/I) and troponin $0.19 \mathrm{ng} / \mathrm{ml}$ (normal $<0.03 \mathrm{ng} / \mathrm{dl}$ ), whereas the rest hematological and biochemical parameters were normal. The chest X-ray was also normal. On the basis of the electocardiographic and cardiac enzyme findings (i.e. elevated troponin, CK-MB) the diagnosis of an AMI was established.

The patient was treated with loading dose of clopidogrel (600 mg), aspirin and heparin (5000 IU). Urgent coronary angiography was performed $(10 \mathrm{~h}$ after the onset of the headache) which showed that the left anterior descending artery was totally occluded, whereas the rest coronary arteries were angiographically normal. Primary percutaneous coronary intervention (PCI) was performed in the culprit lesion followed by zotarolimus-eluting stent implantation. Of note, the headache was totally resolved during the PCI and the patient remained asymptomatic for the rest of his hospitalization. In the follow-up ECG the q waves were preserved, whereas the elevated ST segments and inverted $\mathrm{T}$ waves gradually returned to normal. Cardiac enzymes (CK-MB, troponin) followed a declining course. On the basis of elevated serum glucose and abnormal glucosylated hemoglobin $(\mathrm{HbA} 1 \mathrm{c}=12.6 \%)$ the diagnosis of diabetes mellitus was established and the patient was started on insulin treatment. An echocardiogram 7 days after the onset of the symptoms showed left ventricular dilatation with mildly impaired ejection fraction $(45 \%)$, as well as anteroseptal wall and apex hypokinesis. The patient was discharged from the hospital in good clinical condition on the eighth day of hospitalization. 
$\mathbf{a}$

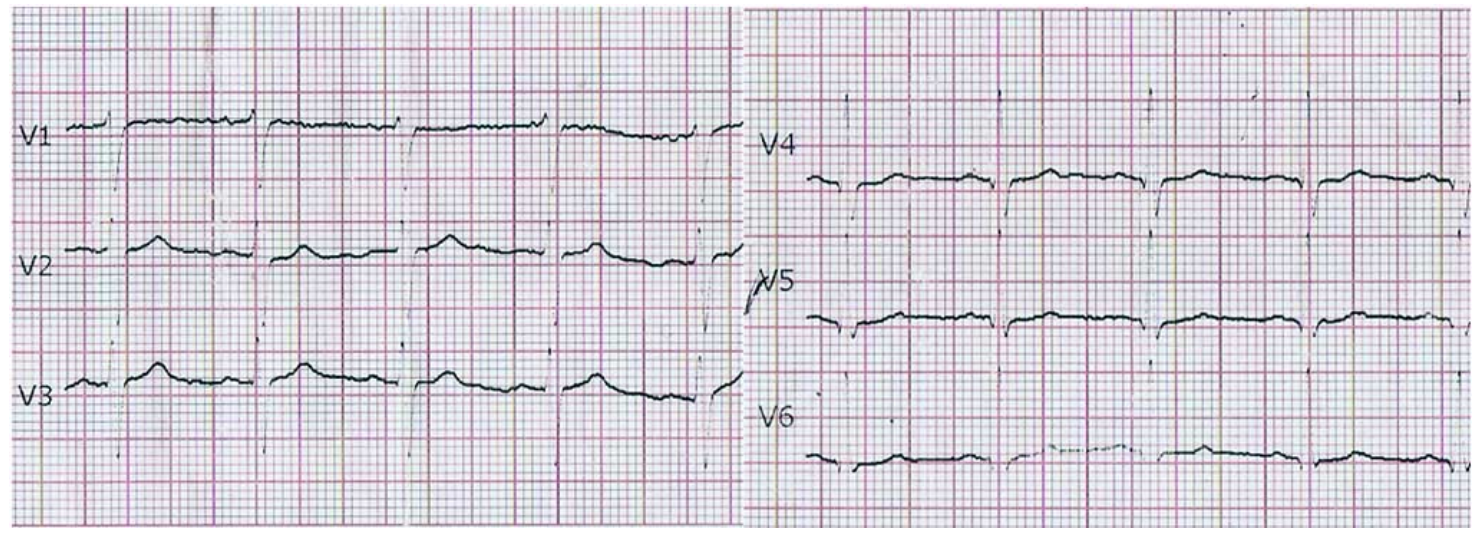

b

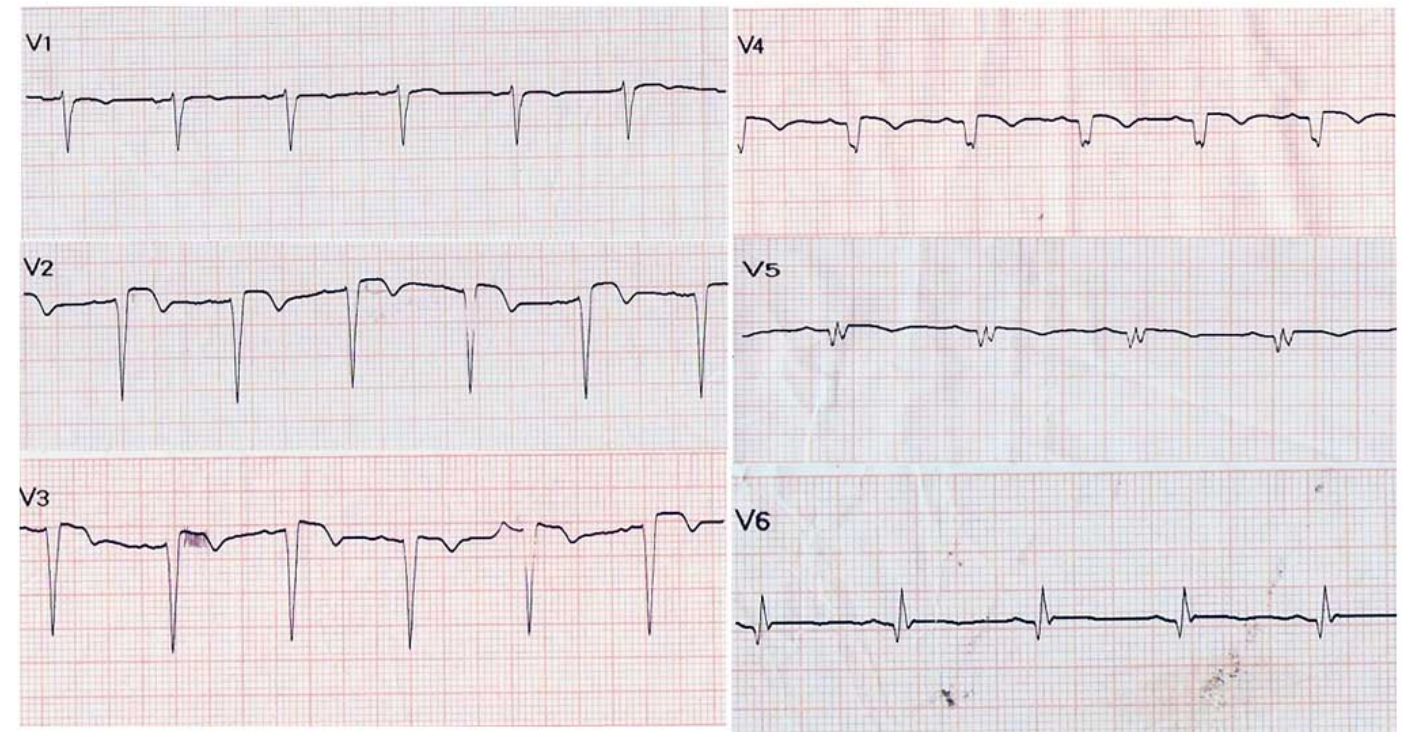

Fig. (1). (a) ECG at $4 \mathrm{~h}$ after the onset of the headache (evaluation in primary health center). (b). ECG at $6 \mathrm{~h}$ after the onset of the headache (admission to the hospital).

\section{DISCUSSION}

The diagnosis of AMI is generally straightforward for most of the patients who present with typical chest pain. However, the diagnostic procedure becomes difficult when the patient complains for atypical symptoms. In most studies headache may co-exist with other typical symptoms. However, headache as a sole leading symptom of an AMI is extremely rare, as only a few reports exist in the literature which mostly refer to patients over 60 years old [3-6].

The presence of risk factors for cardiovascular disease, such as arterial hypertension, diabetes mellitus, hyperlipidemia, smoking, and positive family history increase the possibility of AMI, and therefore AMI should always be included in the differential diagnosis when evaluating such patients. In the current case, the patient was not aware of having diabetes mellitus. Undiagnosed, and therefore, longstanding diabetes mellitus and the subsequent diabetic polyneuropathy was likely associated with the absence of chest pain.

The fact that the patient was relieved from his severe headache immediately after the PCI suggests that the headache and cardiac ischemia may be closely correlated.
Although the underlying pathophysiologic mechanism of headache is not well understood, one could speculate that an acute reduction of the cardiac output as a result of an AMI, especially in the presence of cerebral artery atherosclerosis, could result to the onset of neurological manifestations, such as headache. Alternatively, the headache in the setting of an acute cardiac event may be related to a generalized catecholaminemia-induced vasospastic disorder and subsequent increase of intracranial pressure [3, 8]. Another explanation of the headache in the setting of an AMI involves the perception of cardiac ischemic pain as headache due to the convergence - most likely at a thalamic level - of cardiac autonomic nerve fibers with somatic nerves originating from the head $[3,5,7,8]$. Finally, it has been suggested that headacheeliciting mediators released during cardiac ischemia may also have a role in the manifestation of headache; however this issue is still under investigation [8].

In conclusion, we present an extremely rare case of an AMI presenting with headache as a sole symptom. Physicians should always consider headache as a presenting symptom of an acute coronary event, especially in patients at risk for cardiovascular disease. In a broader spectrum, physicians should consider the possibility of ischemic heart disease at 
every patient with recurrent headache on exertion, after the exclusion of intracranial pathology, especially in the presence of cardiovascular risk factors.

\section{REFERENCES}

[1] Antman Elliot, Braunwauld Eugene: Acute Myocardial Infarction. In Braunwald E, ed. Heart Disease: A Textbook of Cardiovascular Medicine, $6^{\text {th }}$ Ed. Philadelphia, WB Saunders 2001; pp. 1127-9.

[2] Deo R, Christopher P, Cannon, James A. de Lemos: ST Segment Elevation Myocardial Infarction. In: Rosendorff C, Ed. Essential Cardiology Principles and Practice, $2^{\text {nd }}$ ed. Totowa, New Jersey, Humana Press, 2005; pp. 491-2.

[3] Amendo MT, Brown BA, Kossow LB, Weinberg FM. Headache as the sole presentation of acute myocardial infarction in two elderly patients. Am J Geriatr Cardiol 2001; 10: 100-1.
[4] Bowen J, Oppenheim G. Headache as a presentation of angina Reproduction of symptoms during angioplasty. Headache: J Head Face Pain 1993; 33: 238-9.

[5] Auer J, Berent R, Lassnig E, Eber B. Headache as a manifestation of fatal myocardial infarction. Neurol Sci 2001; 22: 396-7.

[6] Sendovski U, Rabkin Y Goldshlak L, Rothmann MG. Should acute myocardial infarction be considered in the differential diagnosis of headache? Eur J Emerg Med 2009; 16: 1-3.

[7] Grace A, Horgan J, Breathnach K, Staunton H. Anginal headache and its basis. Cephalgia 1997; 17: 195-6.

[8] Petersen JA, Nielsen FE. Headache: a rare manifestation of angina pectoris. Ugeskr Laeger 2002; 164: 2515-6. 